Du X et al / Robust SV Benchmark of An Asian using long-sequencing

\title{
1 Robust Benchmark Structural Variant Calls of An Asian Using the 2 State-of-Art Long Fragment Sequencing Technologies
}

4 Xiao Du ${ }^{2,7, \#}$, Lili Li ${ }^{1, \#}$, Fan Liang ${ }^{3, \#}$, Sanyang Liu ${ }^{4, \#}$, Wenxin Zhang ${ }^{1}$, Shuai Sun $^{2,7}$, Yuhui

$5 \quad$ Sun $^{2,8}$, Fei Fan ${ }^{5,8}$, Linying Wang ${ }^{5,8}$, Xinming Liang ${ }^{6}$, Weijin Qiu ${ }^{6}$, Guangyi Fan ${ }^{2,7}$, Ou

6 Wang $^{5,8}$, Weifei Yang ${ }^{4}$, Jiezhong Zhang ${ }^{4}$, Yuhui Xiao ${ }^{3}$, Yang Wang ${ }^{3}$, Depeng Wang ${ }^{3, *}$,

7 Shoufang $\mathrm{Qu}^{1, *}$, Fang Chen ${ }^{5,6, *}$, Jie Huang ${ }^{1, *}$

$9{ }^{1}$ National Institutes for food and drug Control (NIFDC), No.2, Tiantan Xili Dongcheng

10 District, Beijing 10050, China.

$11{ }^{2}$ BGI-Qingdao, BGI-Shenzhen, Qingdao 266555, China

$12{ }^{3}$ GrandOmics Biosciences, Beijing 102200, China.

$13{ }^{4}$ Annoroad Gene Technology (Beijing) Co., Ltd, Beijing 102200, China.

$14{ }^{5}$ BGI-Shenzhen, Shenzhen 518083, China

$15{ }^{6}$ MGI, BGI-Shenzhen, Shenzhen 518083, China.

$16{ }^{7}$ State Key Laboratory of Agricultural Genomics, BGI-Shenzhen, Shenzhen 518083, China

$17{ }^{8}$ China National GeneBank, BGI-Shenzhen, Shenzhen 518120, China.

19 \# These authors contributed equally.

$20 *$ Correspondence authors.

21 Email: jhuang5522@126.com (Huang J), fangchen@ genomics.cn (Chen F),

22 qushoufang@126.com (Qu S), wangdp@grandomics.com (Wang D).

25 Total word counts (from "Introduction" to "Materials and methods"): 5827

26 Total figures: 4

27 Total tables: 2

28 Total supplementary figures: 18

29 Total supplementary tables: 3

30 Total supplementary files: 1

31 Number of all the Reference: 48

32 Number of the Reference from 2014: 28 
Du X et al / Robust SV Benchmark of An Asian using long-sequencing

\section{Abstract}

34 The importance of structural variants (SVs) on phenotypes and human diseases is now 35 recognized. Although a variety of SV detection platforms and strategies that vary in 36 sensitivity and specificity have been developed, few benchmarking procedures are available

37 to confidently assess their performances in biological and clinical research. To facilitate the 38 validation and application of those approaches, our work established an Asian reference 39 material comprising identified benchmark regions and high-confidence SV calls. We 40 established a high-confidence SV callset with 8,938 SVs in an EBV immortalized B 41 lymphocyte line, by integrating four alignment-based SV callers [from 109× PacBio 42 continuous long read (CLR), 22x PacBio circular consensus sequencing (CCS) reads, 104× 43 Oxford Nanopore long reads, and 114x optical mapping platform (Bionano)] and one de novo 44 assembly-based SV caller using CCS reads. A total of 544 randomly selected SVs were 45 validated by PCR and Sanger sequencing, proofing the robustness of our SV calls. 46 Combining trio-binning based haplotype assemblies, we established an SV benchmark for 47 identification of false negatives and false positives by constructing the continuous high 48 confident regions (CHCRs), which cover $1.46 \mathrm{~Gb}$ and $6,882 \mathrm{SVs}$ supported by at least one 49 diploid haplotype assembly. Establishing high-confidence SV calls for a benchmark sample 50 that has been characterized by multiple technologies provides a valuable resource for 51 investigating SVs in human biology, disease, and clinical diagnosis.

52 KEYWORDS: Asian benchmark; Structural variation; Haplotype-resolved; Sanger

53 validation 
Du X et al / Robust SV Benchmark of An Asian using long-sequencing

\section{Introduction}

56 SVs are generally defined as genomic changes spanning at least 50 base pairs (bp), including

57 deletions, insertions, duplications, inversions, and translocations [1]. They contribute to the

58 diversity and evolution of human genomes at individual and population levels [2, 3]. Owing

59 to their large size, SVs often exert greater impacts on gene functions and phenotypic changes

60 than small variants [4-7]. The importance of SVs has been highlighted by their contribution

61 to human diseases including cardiovascular diseases [8], autism [9], and a range of disorders

62 [10]. Therefore, it is crucial to systematically profile SVs in the human genome for both

63 biological research and clinical studies.

64 There are no gold-standard benchmarking procedures for SVs from next-generation

65 sequencing (NGS) platforms. SVs from NGS are largely inferred from indirect evidence of 66 disturbance of read mapping around the variation. Because SVs tend to reside within 67 repetitive DNA and often span more base pairs than short reads ( $<1000 \mathrm{bp}$ ), the short reads of 68 NGS usually lack sensitivity, leading to inevitable challenges in SV detection [11, 12]. 69 Moreover, SV detection approaches vary in both sensitivity and specificity, as they 70 emphasize on different SV-dependent and library-dependent features. Accurate identification 71 of structural variation is very complex that requires characterization of the multifaceted 72 features of SVs, including sequence information, type of variation, length, and location of 73 breakpoints. As a result, different SV callers make inconsistent predictions [12, 13]. 74 Therefore, owing to the complexity of SVs and the inconsistency of different SV callers, a 75 comprehensive assessment of SV detection has been problematic.

76 Several efforts have been made in the community to benchmark SV calls. The Genome in 77 a Bottle Consortium (GIAB) started building high-quality benchmark SV calls by distributing 78 a set of 2,676 high-confidence deletions and 68 high-confidence insertions using SVClassify 79 for a pilot genome (HG001/NA12878) in 2016 [14]. Recently, GIAB released a more 80 comprehensive SV benchmark set for the son (HG002/NA24385) in an Ashkenazi Jewish trio 81 with $2.66 \mathrm{~Gb}$ benchmark regions and 9,641 high-confidence SVs supported by at least one 82 diploid assembly, but the identified SVs were not validated using experimental methods such 83 as Sanger sequencing [15]. A well-characterized SV benchmark set is valuable in identifying 84 false positive and false negative SVs called by various platforms and approaches. Yet, so far 85 we don't have an Asian-specific SV benchmark. The gnomAD-SV that comprises SVs from 8614,891 genomes, revealed that different continental populations exhibited different levels of 
Du X et al / Robust SV Benchmark of An Asian using long-sequencing

87 genetic diversity and SV features [16]. Therefore, designing an Asian benchmark is very necessary for promoting Asian genomic and disease research.

Our work is aimed at designing an Asian reference material comprising identified benchmark regions and high-confidence SV calls. This Asian benchmark is valuable for Asian studies in three aspects. Firstly, it provides material basis for Asian genomic and clinical research. It collects and preserves Asian genetic resources, accessible for Asianspecific biological experiments and drug screening. Secondly, the high-confidence SV calls for a benchmark cell line will serve as a gold-standard for evaluating the performance of a variety of SV detection platforms or strategies, including NGS and long-read sequencing technologies. Thirdly, this set of standards will become a threshold for clinical testing. It helps validate SV detection approaches in clinical practice, and on basis of the design of this benchmark, future benchmark comprising pathogenic SVs will be developed for clinical evaluation of SV detection related to specific diseases.

100 Establishment of immortalized cell lines is a routine strategy for building a reference 101 material for biological research and clinical practice, among which immortalized B

102 lymphocyte line transformed by Epstein-Barr virus (EBV) is a mainstream approach used by 103 the international genetic storage institutions, including the NIGMS Human Genetic Cell 104 Repository and the UK Biobank. EBV infection leads to B lymphocytes proliferation and 105 immortalization in vitro, resulting in establishment of the immortalized B lymphocytes cell 106 lines, which potentially provide unlimited genomic DNA resources and have been 107 extensively used as a biological source for genetic and medical studies [17]. Previous studies 108 and publications suggest EBV exists in the episomal form and is not integrated into the host 109 cell chromosome, maintaining the host genome intact [18-20].

110 The advent of long-read sequencing technologies has greatly aided SV characterization. 111 Although different long-read sequencing platforms applied diverse technologies, they are 112 different from NGS by producing very long reads (1-100kb). In contract to NGS short-read 113 data, the long-read data provide an advantage potential to increase the reliability and 114 resolution of SV detection [21]. Given the advantages of long reads, our work established a 115 high-confidence Asian SV benchmark for deletions and insertions by establishing an EBV 116 immortalized B lymphocyte line and undertaking large-scale SV benchmarking across a 117 range of the latest long-read sequencing or optical mapping techniques, including PacBio 118 continuous long reads (CLR), PacBio circular consensus sequencing (CCS) reads, Oxford 119 Nanopore long reads, and Bionano optical mapping (Figure 1). After comparing the 120 performance of different platforms, we integrated and genotyped the final SV callset. Sanger 
Du X et al / Robust SV Benchmark of An Asian using long-sequencing

121 sequencing validated the high confidence of our SV calls. We assembled haplotype-resolved 122 diploid genomes by a trio binning approach using the PacBio CCS reads, and only high123 confidence SVs supported by at least one diploid haplotype assembly will be included in the 124 SV benchmark. The established cell lines and SV benchmark will provide a standard for 125 assessing the precision and accuracy of different SV detection approaches, and ensure 126 delivering accurate and reliable results for biological and genomic research in Asians. The 127 immortalized B lymphocyte line will serve as an unlimited resource of Asian genomic DNA 128 that can be extensively used as a biological source for future SV and medical studies.

\section{Results}

\section{Sample establishment and sequencing results}

133 Infection with Epstein-Barr virus (EBV) led to B lymphocytes proliferation and 134 subsequent immortalization in vitro. Specifically, peripheral venous blood B lymphocytes of 135 a normal Chinese man from Beijing were infected with EBV and treated with cyclosporine A 136 (CyA) to increase the immortalization efficiency [17]. Morphology of the transformed cells 137 was checked, and transformed cells were passaged and frozen for storage. Resuscitation 138 experiments showed typical cell deformation and clonal growth characteristics. Free from 139 contamination, cells were passaged continuously and grew well after resuscitation. Finally, an 140 immortalized B lymphocyte line (CNGB030001) was successfully established.

141 By sequencing the cells, we generated 312.77Gb ( 104-fold) ONT data, $326.98 \mathrm{~Gb}$ 142 ( 109-fold) PacBio CLR data, and 341.67 Gb ( 114-fold) Bionano data (Table 1). Compared 143 with PacBio CLR, ONT showed a similar sequencing accuracy rate and obviously longer 144 read length (CLR: $9.2 \mathrm{~kb}$ versus ONT: $24.6 \mathrm{~kb}$ ). In addition, we obtained $22 \times$ highly 145 accurate and long PacBio HiFi CCS reads after error correction from $869.48 \mathrm{~Gb}$ raw data 146 ( 290x). The percentage of Q20 (accuracy rate: 99\%) of the total CCS reads was 67.6\% with 147 an average read length of $12.0 \mathrm{~kb}$, providing a high-quality foundation for SV calling. 148 According to read length-GC plots, these four platforms performed very well in terms of 149 uniformity in read length and GC content (Additional file 1: Figures S1-S4).

\section{Candidate SV calls from different sequencing platforms}

152 High accuracy is the prerequisite for establishing SV benchmark. For accuracy concern, we 153 focused on detecting and characterizing large insertions and deletions in this work (Figure 1). 
Du X et al / Robust SV Benchmark of An Asian using long-sequencing

154 By aligning PacBio CLR subreads, we identified 5,871 deletions and 6,936 insertions (Table

155 2). The size distribution of deletions displayed $300 \mathrm{bp}$ and $6 \mathrm{~kb}$ peaks related to SINE-Alu and 156 LINE elements respectively (Additional file 1: Figure S5), suggesting effective SV calling by 157 long reads [22, 23]. Compared to PacBio CLR, 17,901 SVs with 8,317 deletions and 9,584 158 insertions were obtained by aligning PacBio CCS sequencing reads (Table 2, Additional file 159 1: Figure S6). Most of the additional SVs from PacBio CCS were 50 100 bp deletions. 160 Similar to PacBio CLR result, both SINE-Alu and LINE deletions were identified, but no 161 LINE elements for insertions were found in CCS SV calls, probably due to the limitation of 162 read length (Additional file 1: Figure S6).

163 The average read length of ONT data is longer than that of PacBio and the Bionano optical mapping relies on the density of restriction sites on the genome [24], thus theoretically they could efficiently detect the $6 \mathrm{~kb}$ LINE elements for insertions. We detected 14,385 SVs with 7,668 deletions and 6,717 insertions using ONT data, as well as 4,758 SVs by Bionano (Table 2). A $\sim 6 \mathrm{~kb}$ LINE insertion peak was successfully detected by both ONT and Bionano (Additional file 1: Figure S7-S8), but Bionano failed to detect the two short SINE-Alu events for deletions and insertions.

Apart from the alignment strategies, a de novo assembly-based method was applied for SV calling. We performed de novo assembly using 22× PacBio CCS reads, producing 3,542 contigs with the maximum length of $72 \mathrm{Mb}$ and the N50 of $13 \mathrm{Mb}$. A good collinearity was observed from aligning the assembled contigs against the hs37d5 reference genome, indicating no visible structural errors were introduced in the assembly (Additional file 1: Figure S9). Finally, we detected 27,727 SVs using smartie-sv [25], more than those from alignment-based approaches (Table 2). The increase was mainly from small-scale insertions and deletions. Most noteworthy, the expected four insertion and deletion peaks related to SINE-Alu and LINE elements were all observed in the assembly-based SV calls (Additional file 1: Figure S10).

\section{Comparisons among different platforms}

182 None of the approaches is comprehensive in discovering SVs on their own. A significant 183 fraction of the identified variants was unique to a particular approach. The counts of unique 184 SVs and SV overlapping among SV calls from different calling approaches were summarized 185 in Figure 2a. PacBio CLR possessed the least unique variants (491), and CCS assembly186 based SV calls had the most unique variants $(7,930)$. Due to the specificity of Bionano, which 187 is not accurate at base resolution, there were only 160 common SVs shared by all five calling 
Du X et al / Robust SV Benchmark of An Asian using long-sequencing

188 approaches. With Bionano excluded, the three alignment-based single-molecular sequencing

189 (PacBio CLR, PacBio CCS reads and ONT) approaches showed high consistency of SV calls 190 with 8,156 common SVs. After integrating the CCS assembly-based result, the total number 191 of common SVs reached 6,355 for the four datasets.

\section{Construction of high-confidence SV calls}

195 We integrated above candidate SV calls to construct a high-confidence SV callset by following specific steps and criteria (Figure 1). In consideration of the features of different sequencing platforms, there were two main reasons for applying these criteria. First, the outstanding long-read sequencing capacities of ONT, PacBio CLR, and Bionano guaranteed the longest possible read length, facilitating successful cover of large SVs. Second, PacBio CCS reads and CCS assembly approach emphasized on the high accuracy of the SVs. The longest possible read length and high accuracy guaranteed the high confidence of final SV calls. After filtering and integrating, a callset comprising 8,938 high-confidence SVs was established. SV distributions across all autosome chromosomes showed that the number of the distributed SVs had a good linear correlation with the length of the corresponding chromosomes $\left(\mathrm{R}^{2}=0.85\right.$, $\mathrm{p}$-value $<0.0001$, Additional file 1: Figure $\left.\mathrm{S} 11\right)$.

We examined the support for high-confidence SV callset from different sequencing platforms. Bionano showed the lowest support with 250 shared SVs compared to the highconfidence SV calls, and alignment-based CCS displayed the highest support with 8,914 shared SVs. The CCS assembly-based approach (6,419), PacBio CLR (6,797), and ONT $(7,603)$ showed similarly high support (Additional file 1: Figure S12). The length

211 distributions of high-confidence SVs clearly revealed four SINE-Alu and LINE peaks (Figure

212 2c). Moreover, distributions of the insertions and deletions on each chromosome were 213 consistent with the density of SINE-Alu and LINE elements (Additional file 1: Figure 2b, 214 Table S1).

\section{PCR and Sanger sequencing validated high-confidence SVs}

To validate the accuracy of high-confidence SV calls, 400 SVs were randomly selected from 8,938 SVs for PCR amplification, of which $244 \mathrm{SVs}$ were successfully amplified. We next randomly selected a second batch of $200 \mathrm{SVs}$ that contained 56 amplification-failed SVs 
Du X et al / Robust SV Benchmark of An Asian using long-sequencing

221 successfully amplified after PCR primer re-designs. Of the 544 SVs assessed by PCR, 203

222 and 341 are located in the genic and inter-gene regions, respectively. In total, $360 \mathrm{SVs}$ were 223 successfully amplified, and the overall amplification success rate was $66.2 \%$, with 224 amplification rate in genes reaching 90.6\% - notably higher than $51.6 \%$ in inter-gene regions

225 (Figure 3B). This result is not unexpected, as PCR amplification tends to be hindered by 226 complex regions, such as repetitive abundant regions. Moreover, we analysed the length of 227 amplified SVs and found smaller-size SVs had higher amplification rates than that of larger228 size SVs (Additional file 1: Figure S13).

229 Among 360 amplification sites, 317 ( 88.1\%) were successfully sequenced by paired-end 230 Sanger sequencing and aligned to the reference genome (Figure 3A). The sequenced SVs 231 were compared to the high-confidence SVs for evaluation by checking the SV type, SV 232 length, and breakpoint position separately (Additional file 1: Figure S14). The consistency of 233 length or breakpoint position was assessed by stringent matching of coordinate positions 234 within $10 \mathrm{bp}$. Loci due to heterozygosity or low sequencing quality could not be effectively 235 distinguished and were classified as uncertain. For instance, insertions exceeding 500 bp 236 could not be detected by a single Sanger reaction, and thus were classified as uncertain. After 237 excluding the uncertain sites, the concordance of SV type, length, and breakpoint position 238 between Sanger sequenced SVs and our high-confidence SVs reached 93.5\%, 84.3\%, and $23970.5 \%$, respectively (Figure 3C). SVs in genic regions displayed higher concordance of type 240 (97.7\%) and length (91.9\%) than SVs in intergenic regions (89.9\% for type; $76.8 \%$ for length; 241 Figure 3C). While the concordances of SV type and breakpoint position were not influenced 242 by the SV size, the concordance of SV length dropped a little bit as SV size increased 243 (Additional file 1: Figure S15). These results suggest that the high concordance from Sanger 244 sequencing highly supports the robustness of our high-confidence SV calls.

\section{Establishment of the SV benchmark}

With 8,938 high-confidence SV calls, we were aimed to construct a benchmark SV callset that could confidently exclude false positives and false negatives of the tested technology in the benchmark regions. To realize that, we applied a trio-binning based approach using the PacBio CCS data to identify haplotype-resolved SVs via haplotype assemblies, acting as another standard for proofing benchmark SVs. Specifically, using 183.6 Gb (61-fold) shortreads of the subject's father and $184.7 \mathrm{~Gb}$ (62-fold) short-reads of the subject's mother generated by DNBSEQ-G400 sequencing platform, 77.46\% of the subject's PacBio CCS data were unambiguously partitioned into paternal- and maternal- inherited reads using the trio- 
Du X et al / Robust SV Benchmark of An Asian using long-sequencing

255 binning strategy by integrating five different $k$-mers [26] (Additional file 1: Table S2). Then 256 we assembled two haplotypes using the biparental CCS reads by Canu independently [27].

257 The paternal and maternal haplotype assemblies spanned $2.76 \mathrm{~Gb}$ (contig N50 of $726 \mathrm{~kb}$ ) and $2582.92 \mathrm{~Gb}$ (contig N50 of 1,489 kb), respectively. The haplotype assemblies were aligned 259 against human reference genome using blasr (v5.3.3) and SVs were called by smartie-sv 260 independently.

261 On basis of haplotype assemblies, we constructed the continuous high-confidence regions 262 (CHCRs), on which identified SVs should be arbitrarily supported by both the high263 confidence SV calls and the paternal or maternal, haplotype-resolved SVs (Figure 4A). 264 Finally, we identified 4,388 such CHCRs spanning $1.46 \mathrm{~Gb}$ with 6,882 high-confidence SV 265 calls. These 6,882 SV calls constituted our final benchmark SV callset, serving as a gold266 standard containing comprehensive SVs in benchmark genomic regions in Reference 267 Material CNGB030001. In other words, in these 4,388 benchmark regions, we consider only 268 those 6,882 benchmark SVs are expected in sample CNGB030001, which can be used to 269 assess the performance of SV detection from different platforms and approaches.

\section{Comparison to GIAB benchmark}

We compared our Asian benchmark to the recently released GIAB Tier 1 benchmark. Both benchmarks are designed for evaluating the detection of deletions and insertions. Our Asian benchmark comprises 3,346 deletions and 3,536 insertions, in contrast to the GIAB benchmark that includes 4,069 deletions and 5,262 insertions. In total, 3,326 SVs (48.3\%) were shared by these two benchmarks. Comparison of benchmark SVs is dependent on the identified benchmark genome regions. When comparing the benchmark regions, $1.33 \mathrm{~Gb}$ of the $2.51 \mathrm{~Gb}$ benchmark regions in GIAB overlapped with our Asian benchmark regions. Within these overlapping regions, 3,313 SVs (62.4\%) were shared by those two benchmarks, and 1,997 (37.6\%) and 1,785 (35.0\%) unique SVs were possessed by our Asian benchmark and GIAB benchmark, respectively (Additional file 1: Figure S16). This high overlap supports previous observations that many SVs are shared between different individuals [28]. Compared to common SVs, more small-size deletions and more SINE-Alu insertions were identified in Asian-specific SVs (Additional file 1: Figure S17). The unique SVs in overlapping benchmark regions reflect the genetic diversity of different individuals from different continents, which further illustrates the necessity of establishing Asian-specific reference materials and benchmarks. 
Du X et al / Robust SV Benchmark of An Asian using long-sequencing

289

290

291

292

293

294

295

296

297

298

299

300

301

302

303

304

305

306

307

308

309

310

311

312

313

314

315

316

317

318

319

320

321

\section{Application of the benchmark}

The SV benchmark enables evaluation of the performance of different technologies in SV detection. Here we used our 6,882 benchmark SVs to assess the robustness of the three longread sequencing technologies (PacBio CCS, CLR, and ONT) by checking their F1-scores under different sequencing depths. F1-scores of all sequencing platforms increased as their sequencing depth increased. When the sequencing depth reached to $11 \times$, all F1-scores approached their saturation points (CCS: 85.4\%, CLR: $83.0 \%$ and ONT: 82.6\%) (Figure 4B). It should be noted that at higher sequencing depth (20x), PacBio CCS data was the top performer with a higher F1-score of $86.8 \%$ than the other two technologies (CLR: $85.1 \%$ and ONT: $84.5 \%$ ). Our SV benchmark was constructed by integrating SV calls from diverse long fragment sequencing platforms and SVs from diploid assemblies. The F1-score results indicate that none of the three platforms could perfectly detect all SVs in benchmark regions on their own. It is necessary to integrate various approaches and technologies to realize comprehensive and confident SV detection.

We compared our benchmark SVs to the insertions and deletions identified by NGS data, which was generated in another parallel project on the same cell line CNGB030001, to evaluate the performance of NGS platforms in SV detection. We evaluated four representative detection tools including Manta [29], GRIDSS [30], LUMPY [31], and BreakDancer [32] to call SVs from MGISEQ-2000 and NovaSeq 6000 sequencing data, separately. Of the 3,536 insertions in our Asian benchmark, (a) 39.5\% and 6.7\% were detected by Manta and Gridss in MGISEQ data, respectively; (b) $28.3 \%$ and $14.3 \%$ were detected by Manta and Gridss in NovaSeq data, respectively; and (c) Lumpy and Breakdancer were not capable of detecting insertions from NGS data (Additional file 1: Table S3). Of the 3,346 deletions, (a) detection by Manta from MGISEQ and NovaSeq had 56.8\% and 62.8\% sensitivity, respectively; (b) Gridss, Lumpy, and Breakdancer displayed similar sensitivity in MGISEQ ranging from 38.3\% 41.4\% and in NovaSeq ranging from 30.9\% 50.5\% (Additional file 1: Table S3). These results suggest that SVs from NGS platforms differ among detection tools by displaying different sensitivities and specificities, and in general show low sensitivity to the detection of insertions. SV detection capacity also differs by different NGS platforms (MGISEQ-2000 vs. NovaSeq 6000). These results agree with the fact that different detection strategies emphasize on different SV-dependent and librarydependent features, highlighting the need for establishing SV benchmark using long reads. 
Du X et al / Robust SV Benchmark of An Asian using long-sequencing

\section{Discussion}

324 A robust SV benchmark in specified genome regions provides gold-standard for evaluating 325 the performance of various SV detection strategies and platforms in routine and clinical 326 research. To form robust high-confidence SV calls, multiple SV call sets from a variety of

327 methods and sequencing technologies need to be evaluated and integrated. PacBio adopts a 328 sequencing-by-synthesis strategy and produces two types of reads. The continuous long reads 329 (CLR) emphasizes on the longest possible reads, and the circular consensus sequencing (CCS) 330 reads are featured for high accuracy (> 97\%). Oxford Nanopore Technology (ONT) works by 331 monitoring changes to an electrical current as nucleic acids are passed through a protein 332 nanopore. The Bionano linearizes and images long DNA strands that are nicked and 333 fluorescently labeled to produce single molecule physical maps.

334 Our work established 8,938 high-confidence SV calls by combining SV call sets using 335 both alignment-based SV callers and de novo assembly-based SV callers from above state-of336 art long-read sequencing technologies, and applied experiments and haplotype assemblies for 337 validating and establishing a robust benchmark SV callset. Compared to previous SV work, 338 this study collected high-confidence SV calls by incorporating a deeply sequenced new data 339 type, PacBio CCS sequencing, conducted experimental validation, and applied the new 340 assembly approach trio binning for diploid de novo assemblies (combining two parental 341 whole genome sequencing data) to establish robust benchmark SV calls.

342 We established an Asian SV benchmark for identifying both false negatives and false 343 positives in specified benchmark regions with a well-characterized set of haplotype-resolved 344 SVs. The final benchmark SV callset comprising 6,882 SVs is highly robust. Firstly, it was 345 established on basis of 8,938 high-confidence SVs, which were constructed by integrating 346 comprehensive information from the state-of-art long-read sequencing technologies. 347 Different sequencing platforms and analysis approaches (alignment-based and assembly348 based) complemented each other and an integration of them was robust in detecting confident 349 SVs. All SVs in the high-confidence set had support in reads from more than one technology. 350 Secondly, we validated randomly selected SVs using PCR amplification and Sanger 351 sequencing, confirming the high confidence of our SV calls. Lastly, we took use of trio 352 binning-based haplotype assemblies to distinguish paternal or maternal SVs. Only haplotype353 resolved, high-confidence SVs could be included in the benchmark SV calls. The established

354 Asian benchmark spans 1.46Gb and covers 6,882 SVs supported by at least one diploid 
Du X et al / Robust SV Benchmark of An Asian using long-sequencing

355 haplotype assembly, allowing the community to confidently evaluate the detection capacity 356 for insertions and deletions in future practices.

357 It should be noted that high accuracy is a prerequisite for establishment of the benchmark. 358 Therefore, our established benchmark only covered specific regions of the genome with 359 confirmed accuracy, not the whole genome. In those regions we confirmed that benchmark 360 SVs were highly confident. Our benchmark did not focus on complex SVs (e.g., inversions, 361 duplications, and translocations) either. Importantly, we emphasize that this SV benchmark 362 allows the community to confidently evaluate the performance of various platforms and 363 approaches in detecting insertions and deletions. The deeply sequenced data in this study can 364 be used in future work to extend our understanding of complex SVs. As mentioned above, in 365 another parallel project [33] we generated about 4.16T clean data of the same cell line using 366 seven sequencing strategies in different laboratories, including two BGI regular NGS 367 platforms, three Illumina regular NGS platforms, single tube long fragment read (stLFR) 368 sequencing, and 10X Genomics Chromium linked-read sequencing. Those large data will 369 provide comprehensive variants information, serving as valuable genomic resources 370 facilitating future genomic or medical research.

371 By analysing extensive SV calls generated from different platforms and calling tools, we 372 found that different technologies had distinct strengths and weaknesses. PacBio CCS reads 373 detected $\sim 5000$ more SVs than PacBio CLR, but neither of them identified the $6 \mathrm{~kb}$ LINE 374 insertions using the alignment-based strategy. CCS assembly-based approach successfully 375 identified four SINE/Alu and LINE elements in insertions and deletions, and detected the 376 largest SV callset with more small-size insertions and deletions. Bionano mapping is based 377 on optical ultra-long single molecules of DNA that are fluorescently labelled at specific 378 restriction sites [24]. Due to its dependency on the density of restriction sites, it failed to 379 accurately detect small-size SVs with the least SVs identified. While other techniques 380 detected more insertions than deletions, ONT was more sensitive to detect deletions than 381 insertions. It could also effectively detect the $6 \mathrm{~kb}$ LINE element insertions.

382 The established reference material CNGB030001 serves as an unlimited resource of Asian 383 genomic resources, facilitating future Asian SV and medical studies. EBV transformed cell 384 lines are widely used internationally in routine and clinical research. Usually cell line genome 385 is relatively stable under a certain number of passages, and after long-term passages genomic 386 instability is a common problem in immortalized cell lines, such as tumor cell lines. In our 387 project, genomic instability after long-term passages wouldn't be a concern for our cell line 388 applications. To release as reference material, we have generated a large quantity of tubes at 
Du X et al / Robust SV Benchmark of An Asian using long-sequencing

389 one time to confirm usage for several years, ensuring low cell passages. Good cell bank management could effectively ensure low cell generations, and regular cell line identifications will help verify the cell line stability. Therefore, cell line CNGB030001 can be widely used for Asian genomic and medical research as a valuable reference material.

\section{Conclusion}

396 Taking advantage of multiple long fragment sequencing platforms, our work established an 397 Asian reference material and developed a robust SV benchmark. PCR amplification and 398 Sanger sequencing were conducted to validate the high quality of our high-confidence SVs. 399 Haplotype assemblies via trio binning were used for identifying haplotype-resolved SVs to 400 construct the final robust benchmark. The performance of SV calling of different 401 technologies across various sequencing depths provides valuable information for further SV 402 studies. Finally, we report that the established benchmark cell line provides valuable Asian 403 genomic resources in biological and medical research and the SV benchmark can serve as a 404 gold standard for benchmarking SV detection approaches in clinical practice.

\section{Materials and methods}

\section{Establishment of immortalized B lymphocyte line}

409 B lymphocyte immortalization was performed according to the published protocol [34] with 410 slight modifications. $4.5 \mathrm{~mL}$ whole blood was collected from a Chinese donor with sodium 411 citrate anticoagulant blood collection tube (369714, Becton Dickinson, New Jersey, US). 412 Peripheral blood mononuclear cells (PBMCs) were isolated from the whole blood by Ficoll 413 density gradient centrifugation (p-05824, GE Healthcare, Illinois, US). The lymphocytes 414 were simulated and transformed by treating cyclosporin A (No.12088, Cayman Chemical, 415 Michigan, US) and EBV that was prepared from collecting the supernatant of B95-8 cells 416 (ATCC CRL-1612). The transformed lymphocytes were monitored by microscope for the 417 performance of transformation. After transformation, the immortalized lymphocytes were 418 cultured on large scale and then divided into $1 \times 10^{6}$ per tube for long-term storage.

419 DNA extraction, library preparation, and sequencing

420 DNA extraction for PacBio and Oxford nanopore 
Du X et al / Robust SV Benchmark of An Asian using long-sequencing

$4215 \times 10^{6}$ frozen cells were suspended in $1 \times$ PBS buffer to reach a total volume of $2 \mathrm{ml}$, and 1

422 volume of ice-cold cell lysis buffer (1.28 M sucrose, $40 \mathrm{mM}$ Tris hydrochloride, $20 \mathrm{mM}$

$423 \mathrm{MgCl}_{2}, 4 \%$ Triton $\mathrm{X}-100$ [pH 7.5]) and 3 volumes of ice-cold distilled water were added. The

424 mixture was incubated for 10 minutes on ice, and nuclear pellets were collected by 425 centrifugation $\left(6,000 \mathrm{rpm}, 5 \mathrm{~min}, 4^{\circ} \mathrm{C}\right)$. The nuclei were completely resuspended in 426 extraction buffer (0.8M guanidine hydrochloride, 30mM Tris, 30mM EDTA, 5\% Tween-20, $4270.5 \%$ Triton-100 [pH 8.0]) containing $1 \%$ sodium dodecyl sulphate (SDS) and proteinase K 428 (2 $\mathrm{mg} / \mathrm{ml}$ final concentration), and incubated at $56^{\circ} \mathrm{C}$ for 2 hours. gDNAs were extracted by 429 phenol-chloroform-isoamyl alcohol (25:24:1 by volume) and chloroform-isoamyl alcohol 430 (24:1 by volume), and precipitated with 0.7 volume of isopropyl alcohol at $-20^{\circ} \mathrm{C}$ for 40 431 minutes. The DNA precipitates were washed in ice-cold $80 \%$ ethanol for twice, collected by 432 centrifugation $\left(12,000 \mathrm{rpm}, 15 \mathrm{~min}, 4^{\circ} \mathrm{C}\right)$, dried under vacuum, and finally resuspended in $433100 \mathrm{ul}$ of EB (10 mM Tris hydrochloride [pH 8.0]). To acquire high-quality DNA, an 434 additional step of purification was performed right after DNA extraction by 0.8 volume of 435 magnet beads from AMPure XP kit (\#A63882, Agencourt) according to the manufacturer's 436 instructions. Agilent 4200 Bioanalyzer (Agilent Technologies, Palo Alto, California) was 437 used to detect the integrity of gDNA. $8 \mu \mathrm{g}$ of gDNA was sheared using g-Tubes (Covaris) 438 and concentrated with the AMPure PB magnetic beads.

439 Library construction and sequencing of PaBio CLR

440 We used a Pacific Biosciences SMRT bell template prep kit 1.0 to construct each SMRT bell 441 library by following the manufacturer's instructions. The constructed libraries were size442 selected on a BluePippin ${ }^{\mathrm{TM}}$ system for molecules $\geq 20 \mathrm{~Kb}$, followed by primer annealing and 443 the binding of SMRT bell templates to polymerases with the DNA/Polymerase Binding Kit. 444 Finally, sequencing was performed on the Pacific Bioscience Sequel platform (Annoroad 445 Gene Technology Co., Ltd China) for $10 \mathrm{~h}$ and finally sequenced by CLR mode with the 446 Sequel System (Pacific Biosciences, CA).

447 Library construction and sequencing of PacBio CCS

448 SMRTbell libraries were prepared using the 'Express Template Prep Kit 1.0' protocol 449 (Pacific Biosciences, CA). 5 ug genomic DNA was sheared to $\sim 15 \mathrm{~kb}$ fragments by a $\mathrm{g}$ 450 TUBE (\#520079, Covaris) centrifugation (2,000 Xg, 2 min, twice), was size-selected for 10 $451 \mathrm{~kb}$ using the BluePippin system (Sage Science, MA) by marker (0.75\% DF Marker S1 High452 Pass 6-10kb vs3) for the 10-20 kb DNA target fragments. Quality control of the libraries was 453 performed by Qubit fluorometer (Life Technologies, CA) and Bioanalyzer 2100 (Agilent, 454 CA). The prepared library was loaded into SMRT cell 1M by Sequel Binding Kit 3.0 (Pacific 
Du X et al / Robust SV Benchmark of An Asian using long-sequencing

455 Biosciences, CA) and finally sequenced by CCS mode with the Sequel System (Pacific 456 Biosciences, CA).

457 Library construction and sequencing of Oxford nanopore

458 Genomic DNA libraries were prepared using the Ligation sequencing 1D kit (SQK-LSK109, 459 Oxford Nanopore Tech., UK). End-repair and dA-tailing of DNA fragments were performed 460 using the Ultra II End Prep module (\#E7546, NEB) by following protocol recommendations. 461 The dA tailed sample was tethered to 1D adapter by Quick Ligation Module (\#E6056, NEB). 462 Finally, the prepared DNA library was loaded into FLO-PRO002 flow cell and sequenced on 463 PromethION (Oxford Nanopore Tech., UK).

464 DNA extraction and sequencing for Bionano

465 The isolation of high-molecular-weight gDNA from immortalized B lymphocyte line was 466 performed with the Bionano Prep ${ }^{\mathrm{TM}}$ Cell Culture DNA Isolation Kit according to the standard 467 protocol of Bionano Prep Cell Culture DNA Isolation Protocol (Document Number: 30026). 468 Sequence-specific labelling of megabase gDNA for Bionano mapping was conducted by 469 nicking, labelling, repairing, and staining (NLRS) following the standard protocol of Bionano 470 Prep $^{\mathrm{TM}}$ Labelling-NLRS. The labelled gDNA was transferred into Bionano Genomics 471 Saphyr ${ }^{\mathrm{TM}}$ for scanning to obtain the optical map.

472 SV calling based on different platforms and methods

473 Alignment-based SV calling

474 (a) For CLR reads, BAM files of CLR reads were exported from SMRT Link (v6.0.0.47841), 475 and aligned to the reference genome (hs37d5) using minimap2 (v2.15-r906-dirty) [35] with 476 parameters “align_opts = "-x map-pb -a --eqx -L -O 5,56 -E 4,1 -B 5 --secondary=no 477 z 400,50 -r 2k -Y -R "@RGItID:rg1altSM:human"'”. (b) For CCS reads, CCS reads BAM 478 files were aligned to the reference genome (hs37d5) using minimap2 (v2.15-r906-dirty) [35] 479 with parameters "-R -t 2 --MD -Y -L -a -x map-pb". According to the mapping positions, 480 SAMtools (v0.1.19) [36] was used to sort the alignments with default parameters. To identify 481 SV, pbsv (v2.1.1) [37] with default parameters was used to sort alignment files. (c) For ONT 482 reads, reads with quality score $>7$ were aligned to the reference genome (hs37d5) using 483 ngmlr (v0.2.7) [38] with the parameter “--presets nanopore”. SVs were called using sniffles 484 (version 1.0.8) [38] with parameters “--min_support 1 --threads 8 --num_reads_report -1 485 genotype". (d) For Bionano data, Bionano data was generated from enzyme BspQI and SVs 486 were called using Bionano Solve pipeline (v3.1) [39] with default parameters.

487 De novo assembly-based SV calling 
Du X et al / Robust SV Benchmark of An Asian using long-sequencing

488 Falcon (v0.3.0) [40] was used for assembly and contigs were aligned to hs37d5 reference 489 genome using blasr (v5.3.3) [41]. SV calling was performed with smartie-sv [25, 42].

\section{Integration of the high-confidence SV calls}

491 The high-confidence SV callsets were integrated from all candidate SV callsets by the 492 following processes: (a) The same type of SVs within $1 \mathrm{~kb}$ with sequence change <20\% were 493 merged into a single SV using SVmerge (v1.2r27) [43]. (b) All SVs detected by PacBio CCS 494 Reads and supported by either PacBio CLR or ONT were retained. SVs longer than $1.5 \mathrm{~kb}$ 495 supported by both PacBio CCS assembly and Bionano mapping were also retained. (c) SVs 496 located in centromeres, telomeres, segmental duplications, and short tandem repeat regions 497 were removed according to the SV annotations by annovar (v20160201) [44]. (d) Hawkeye 498 (v2.0) [45] was used for SV visualization by automatically outputting images for manual 499 checking.

\section{SV validation by PCR amplification and Sanger sequencing}

501 We performed the Sanger validation for two batches of randomly selected SVs. An 502 amplification was considered successful if a clear single band was observed or the expected 503 size band could be purified and separated by gel cutting. The failed SVs showed ambiguous 504 bands. To evaluate the effect of primer design, failed SVs from the first batch were repeated 505 for PCR amplification in the second batch. The corresponding primers were designed with 506 Primer3 by default parameters [46]. Amplification results for each amplicon were validated by electrophoresis and the products were loaded onto 3730 sequencers with pair-end 508 sequencing mode (Thermofisher, Massachusetts, US). Raw sequencing results were analysed 509 by Sequence Scanner Software v2.0 and the low-quality parts were trimmed. The clean reads 510 were mapped to the reference genome hs37d5 by BLAST, and the mapping results were 511 manually checked for SVs. For manual curation, the following criteria were used to evaluate 512 the accuracy of previous SV calls: 1) if there was an SV event supported by any single sanger 513 read; 2) if a previously called SV could match a Sanger call within 10-bp difference in size;

514 and 3) if the breakpoint of a previously called SV could match that of a Sanger call within 10 515 bp differences.

\section{Construction of diploid haplotype genomes using trio binning}

517 Short reads from the parents were used to identify $k$-mers unique to each parent and partition

518 ("trio binning") the CCS reads. The trio binning pipeline was applied to partition paternal and 519 maternal CCS reads [26, 47] using five different $k$-mers including 21 bp (previously reported 520 for trio binning) and longer $k$-mers of 41, 51, 61 and 81 bp. To realize accurate partition, one 521 integration method was used following two criteria: for one CCS read, (a) at least two 
Du X et al / Robust SV Benchmark of An Asian using long-sequencing

522 different k-mers support the same parental source; (b) more than half of the different k-mers 523 support the same parental source.

524 To obtain paternal and maternal haplotype genomes, we used canu (v1.8-r9528) [27] to 525 assemble paternal and maternal CCS reads with parameters "-trim-assemble 526 genomeSize $=3100 \mathrm{~m}$ correctedErrorRate=0.039 -pacbio-corrected", separately. Meanwhile, 527 unassigned CCS reads were used in both two assemblies. Lastly, the paternal and maternal 528 haplotype assemblies were aligned against human reference genome using blasr (v5.3.3) and 529 SVs were called by smartie-sv independently.

530 SV benchmark construction, comparison, and application

531 To establish the benchmark SV callset, we identified the continuous high-confident regions 532 by combining 8,938 high-confidence SVs and SVs called from haplotype-solved assemblies. 533 To evaluate the capability of different platforms, SV calls detected by different technologies 534 were all converted into VCF formats and evaluated against the continuous high-confident 535 regions using Truvari (v1.3) [48] with default parameters. To compare our Asian benchmark 536 to GIAB benchmark, we used SVmerge (v1.2r27) with default parameter “-d 1000” for SV 537 positions, “-1 0.5 ” for SV length difference, and “-r 0.5 ” for SV overlap. Overlapping and 538 unique SVs were enriched for gene pathways by R package clusterProfiler.

539 SVs from two NGS platforms (MGISEQ-2000 and NovaSeq 6000, triplicat calls per sample)

540 were called using four tools and hs $37 \mathrm{~d} 5$ reference genome with corresponding parameters:

541 GRIDSS (default parameters), LUMPY (default parameters), Manta

542 (minCandidateSpanningCount=3, minScoredVariantSize $=50$, minDiploidVariantScore $=10$,

543 minPassDiploidVariantScore $=20, \operatorname{minPassDiploidGTScore~}=15$, $\operatorname{minSomaticScore}=10$,

544 minPass SomaticScore $=30$, useOverlapPairEvidence $=0$,

545 enableRemoteReadRetrievalForInsertionsInGermlineCallingModes $=1$,

546 enableRemoteReadRetrievalForInsertionsInCancerCallingModes $=0$ ), and BreakDancer

547 (num:10001, lower:78.10, upper:465.35, mean:254.28, std:48.32, SWnormality:-31.28). For

548 each sample, $3 \mathrm{SV}$ calls were integrated into a final call by SURVIVOR using following

549 parameters: 1000211030 . We used SVmerge (v1.2r27) with default parameter "-d 1000"

550 for SV positions, “-1 0.5 ” for SV length difference, and "-r 0.5” for SV overlap. To find

551 common SVs between NGS SV results and our SV benchmark.

553 Acknowledgments

554 We thank Inge Seim for reading and offering very helpful suggestions on the writing of this 555 manuscript. 
Du X et al / Robust SV Benchmark of An Asian using long-sequencing

\section{Authors' contributions}

$558 \mathrm{JH}, \mathrm{DW}$, and FC conceived the project. JH, OW, GF, SQ, FL, and FC supervised the study. $559 \mathrm{JH}, \mathrm{LL}, \mathrm{WZ}$, and JZ contributed to sample establishment. FL, SL, SS, YS, XL, WQ, WY, JZ, 560 YX, and YW performed bioinformatics analyses. FF, OW, LW, FC, and SQ performed PCR 561 amplification and Sanger sequencing for evaluation of structural variation. JH, XD, SS, GF, 562 and OW wrote the manuscript with help from all co-authors.

\section{Funding}

565 This work was supported by grants from the National Key Research and Development 566 Program of China (No. 2017YFC0906501).

\section{Availability of data and materials}

569 The sequencing data (including ONT, PacBio CCS, and PacBio CLR data) has been

570 deposited in the CNGB under accession number CNP0000091. Bionano data can be 571 accessible at CNGB FTP site: http://ftp.cngb.org/pub/CNSA/data1/CNP0000091/.

\section{Ethics approval and consent to participate}

574 The experimental procedures were in accordance with the guidelines approved by the 575 institutional review board on bioethics and biosafety of BGI (IRB-BGI). The experiment was 576 authorized by IRB-BGI (under NO. FT19038), and the review procedures in IRB-BGI meet 577 good clinical practice (GCP) principles.

\section{Consent for publication}

580 Not applicable.

\section{Competing interests}

583 The authors have declared no competing interests.

\section{References}

586 [1] Feuk L, Carson AR, Scherer SW. Structural variation in the human genome. Nature Review 587 Genetics 2006;7:85-97. 


\section{Du X et al / Robust SV Benchmark of An Asian using long-sequencing}

588 [2] Sudmant PH, Mallick S, Nelson BJ, Hormozdiari F, Krumm N, Huddleston J, et al. Global

589 diversity, population stratification, and selection of human copy-number variation. Science

$590 \quad 2015 ; 349: a a b 3761$.

591 [3] Sudmant PH, Rausch T, Gardner EJ, Handsaker RE, Abyzov A, Huddleston J, et al. An integrated

592 map of structural variation in 2,504 human genomes. Nature 2015;526:75-81.

593 [4] Chaisson MJP, Sanders AD, Zhao X, Malhotra A, Porubsky D, Rausch T, et al. Multi-platform

594 discovery of haplotype-resolved structural variation in human genomes. Nature Communications

$5952019 ; 10: 1784$.

596 [5] Chiang C, Scott AJ, Davis JR, Tsang EK, Li X, Kim Y, et al. The impact of structural variation on

597 human gene expression. Nat Genet 2017;49:692-9.

598 [6] Pang AW, MacDonald JR, Pinto D, Wei J, Rafiq MA, Conrad DF, et al. Towards a comprehensive 599 structural variation map of an individual human genome. Genome Biol 2010;11:R52.

600 [7] Sebat J, Lakshmi B, Troge J, Alexander J, Young J, Lundin P, et al. Large-scale copy number

601 polymorphism in the human genome. Science 2004;305:525-8.

602 [8] Merker JD, Wenger AM, Sneddon T, Grove M, Zappala Z, Fresard L, et al. Long-read genome

603 sequencing identifies causal structural variation in a Mendelian disease. Genet Med 2018;20:159-63.

604 [9] Sebat J, Lakshmi B, Malhotra D, Troge J, Lese-Martin C, Walsh T, et al. Strong association of de 605 novo copy number mutations with autism. Science 2007;316:445-9.

606 [10] Mantere T, Kersten S, Hoischen A. Long-Read Sequencing Emerging in Medical Genetics.

607 Frontiers in Genetics 2019;10:426.

608 [11] Teo SM, Pawitan Y, Ku CS, Chia KS, Salim A. Statistical challenges associated with detecting

609 copy number variations with next-generation sequencing. Bioinformatics 2012;28:2711-8.

610 [12] Guan P, Sung W-K. Structural variation detection using next-generation sequencing data: A

611 comparative technical review. Methods 2016;102:36-49.

612 [13] Kosugi S, Momozawa Y, Liu X, Terao C, Kubo M, Kamatani Y. Comprehensive evaluation of

613 structural variation detection algorithms for whole genome sequencing. Genome Biol 2019;20:117.

614 [14] Parikh H, Mohiyuddin M, Lam HY, Iyer H, Chen D, Pratt M, et al. svclassify: a method to

615 establish benchmark structural variant calls. BMC Genomics 2016;17:64.

616 [15] Zook JM, Hansen NF, Olson ND, Chapman L, Mullikin JC, Xiao C, et al. A robust benchmark

617 for detection of germline large deletions and insertions. Nature Biotechnology 2020.

618 [16] Collins RL, Brand H, Karczewski KJ, Zhao X, Alföldi J, Francioli LC, et al. A structural

619 variation reference for medical and population genetics. Nature 2020;581:444-51.

620 [17] Hui-Yuen J, McAllister S, Koganti S, Hill E, Bhaduri-McIntosh S. Establishment of Epstein-Barr 621 virus growth-transformed lymphoblastoid cell lines. J Vis Exp 2011.

622 [18] Hussain T, Mulherkar R. Lymphoblastoid Cell lines: a Continuous in Vitro Source of Cells to 623 Study Carcinogen Sensitivity and DNA Repair. Int J Mol Cell Med 2012;1:75-87.

624 [19] Sie L, Loong S, Tan EK. Utility of lymphoblastoid cell lines. J Neurosci Res 2009;87:1953-9. 


\section{Du X et al / Robust SV Benchmark of An Asian using long-sequencing}

625 [20] Thorley-Lawson DA, Gross A. Persistence of the Epstein-Barr virus and the origins of associated 626 lymphomas. N Engl J Med 2004;350:1328-37.

627 [21] Luan M-W, Zhang X-M, Zhu Z-B, Chen Y, Xie S-Q. Evaluating Structural Variation Detection

628 Tools for Long-Read Sequencing Datasets in Saccharomyces cerevisiae. Frontiers in Genetics $6292020 ; 11$.

630 [22] Kim S, Cho C-S, Han K, Lee J. Structural variation of Alu element and human disease.

631 Genomics Informatics 2016;14:70.

632 [23] Sheen F-m, Sherry ST, Risch GM, Robichaux M, Nasidze I, Stoneking M, et al. Reading

633 between the LINEs: human genomic variation induced by LINE-1 retrotransposition. Genome Res

634 2000;10:1496-508.

635 [24] Chen P, Jing X, Ren J, Cao H, Hao P, Li X. Modelling BioNano optical data and simulation

636 study of genome map assembly. Bioinformatics 2018;34:3966-74.

637 [25] Kronenberg ZN, Fiddes IT, Gordon D, Murali S, Cantsilieris S, Meyerson OS, et al. High-

638 resolution comparative analysis of great ape genomes. Science 2018;360:eaar6343.

639 [26] Koren S, Rhie A, Walenz BP, Dilthey AT, Bickhart DM, Kingan SB, et al. De novo assembly of

640 haplotype-resolved genomes with trio binning. Nat Biotechnol 2018;36:1174-82.

641 [27] Koren S, Walenz BP, Berlin K, Miller JR, Bergman NH, Phillippy AM. Canu: scalable and

642 accurate long-read assembly via adaptive k-mer weighting and repeat separation. Genome Res

643 2017;27:722-36.

644 [28] Audano PA, Sulovari A, Graves-Lindsay TA, Cantsilieris S, Sorensen M, Welch AE, et al.

645 Characterizing the Major Structural Variant Alleles of the Human Genome. Cell 2019;176:663-75.e19.

646 [29] Chen X, Schulz-Trieglaff O, Shaw R, Barnes B, Schlesinger F, Källberg M, et al. Manta: rapid

647 detection of structural variants and indels for germline and cancer sequencing applications.

648 Bioinformatics 2016;32:1220-2.

649 [30] Cameron DL, Schröder J, Penington JS, Do H, Molania R, Dobrovic A, et al. GRIDSS: sensitive

650 and specific genomic rearrangement detection using positional de Bruijn graph assembly. Genome

651 research 2017;27:2050-60.

652 [31] Layer RM, Chiang C, Quinlan AR, Hall IM. LUMPY: a probabilistic framework for structural

653 variant discovery. Genome Biol 2014;15:R84.

654 [32] Chen K, Wallis JW, McLellan MD, Larson DE, Kalicki JM, Pohl CS, et al. BreakDancer: an

655 algorithm for high-resolution mapping of genomic structural variation. Nature methods 2009;6:677-81.

656 [33] Huang C, Shao L, Qu S, Rao J, Cheng T, Cao Z, et al. An integrated Asian human SNV and indel

657 benchmark combining multiple sequencing methods. bioRxiv 2019:759043.

658 [34] Yu X, McGraw PA, House FS, Crowe Jr JE. An optimized electrofusion-based protocol for

659 generating virus-specific human monoclonal antibodies. J Immunol Methods 2008;336:142-51.

660 [35] Li H. Minimap2: pairwise alignment for nucleotide sequences. Bioinformatics 2018;34:3094-100. 


\section{Du X et al / Robust SV Benchmark of An Asian using long-sequencing}

661 [36] Li H, Handsaker B, Wysoker A, Fennell T, Ruan J, Homer N, et al. The sequence alignment/map 662 format and SAMtools. Bioinformatics 2009;25:2078-9.

663 [37] Duchesne P, Turgeon J. FLOCK: a method for quick mapping of admixture without source 664 samples. Molecular Ecology Resources 2009;9:1333-44.

665 [38] Sedlazeck FJ, Rescheneder P, Smolka M, Fang H, Nattestad M, von Haeseler A, et al. Accurate 666 detection of complex structural variations using single-molecule sequencing. Nat Methods 667 2018;15:461-8.

668 [39] Shelton JM, Coleman MC, Herndon N, Lu N, Lam ET, Anantharaman T, et al. Tools and 669 pipelines for BioNano data: molecule assembly pipeline and FASTA super scaffolding tool. BMC 670 Genomics 2015;16:734.

671 [40] Chin C-S, Peluso P, Sedlazeck FJ, Nattestad M, Concepcion GT, Clum A, et al. Phased diploid 672 genome assembly with single-molecule real-time sequencing. Nat Methods 2016;13:1050.

673 [41] Chaisson MJ, Tesler G. Mapping single molecule sequencing reads using basic local alignment 674 with successive refinement (BLASR): application and theory. BMC Bioinformatics 2012;13:238.

675 [42] Zhang G, Cai L, Li Q, Bo L, Larkin DM, Lee C, et al. Comparative genomics reveals insights 676 into avian genome evolution and adaptation. Science 2014;346:1311.

677 [43] Wong K, Keane TM, Stalker J, Adams DJ. Enhanced structural variant and breakpoint detection 678 using SVMerge by integration of multiple detection methods and local assembly. Genome Biol $679 \quad 2010 ; 11: R 128$.

680 [44] Wang K, Li M, Hakonarson H. ANNOVAR: functional annotation of genetic variants from high681 throughput sequencing data. Nucleic Acids Res 2010;38:e164-e.

682 [45] Schatz MC, Phillippy AM, Shneiderman B, Salzberg SL. Hawkeye: an interactive visual 683 analytics tool for genome assemblies. Genome Biol 2007;8:R34.

684 [46] Untergasser A, Cutcutache I, Koressaar T, Ye J, Faircloth BC, Remm M, et al. Primer3-new 685 capabilities and interfaces. Nucleic Acids Res 2012;40:e115-e.

686 [47] Li R, Zhu H, Ruan J, Qian W, Fang X, Shi Z, et al. De novo assembly of human genomes with 687 massively parallel short read sequencing. Genome Research 2010;20:265-72.

688 [48] Conte MA, Gammerdinger WJ, Bartie KL, Penman DJ, Kocher TD. A high quality assembly of 689 the Nile Tilapia (Oreochromis niloticus) genome reveals the structure of two sex determination 690 regions. Bmc Genomics 2017;18:341.

\section{$692 \quad$ Figure legends}

693 Figure 1 Workflow of establishing the benchmark SV set integrating different long-read 694 sequencing technologies and approaches

695 Figure 2 Comparisons among candidate SV call sets from different technologies and 696 characterization of the high-confidence SV set 
Du X et al / Robust SV Benchmark of An Asian using long-sequencing

697 A. The number of SVs overlapping between candidate call sets from multiple approaches. B.

698 A circos plot representing SV and repeat number in high-confidence SV calls using sliding

699 non-overlapping windows of $1 \mathrm{Mb}$ length through the human genome. From the outer circle to

700 the inner circle, the four circles represent the number of DEL, INS, SINE/Alu, LINE per 1

$701 \mathrm{Mb}$ window, respectively. c Size distribution for insertions and deletions in the high-

702 confidence SV set.

703 Figure 3 PCR and Sanger sequencing validated the SVs

704 A. An example of Sanger sequencing validating a deletion event on chromosome 8. B. PCR

705 amplification rates for different genomic regions. C. Consistency rates of SV type, SV length,

706 and SV position between Sanger sequenced variants and identified structural variants.

707 Figure 4 Assess the SV calling performance of different technologies

708 A. Construction of continuous confident regions based on two haplotype-resolved diploid

709 SV callsets. B. Evaluation of different sequencing techniques and sequencing depth using the

710 SV callsets.

\section{Tables}

713 Table 1 Summary of sequencing results for different platforms

714 Table 2 Insertion and deletion variation for different calling approaches

\section{Supplementary material}

717 Additional file 1: Supplementary figures and tables.

718 Figure S1 The length and GC content distributions of sequencing reads produced from

719 PacBio continuous long read (CLR) technology

720 Figure S2 The quality of reads varies with the number of sequencing passes produced from

721 PacBio circular consensus sequencing (CCS) technology

722 Figure $\mathbf{S 3}$ The length and GC content distributions of sequencing reads produced from

723 PacBio CCS technology

724 Figure S4 The length and GC content distributions of sequencing reads produced from

725 Oxford Nanopore technology (ONT)

726 Figure S5 The size distributions for 0 1000bp and 1000 10000bp ranges for candidate SV

727 calls from PacBio CLR

728 Figure S6 The size distributions for 0 1000bp and 1000 10000bp ranges for candidate SV

729 calls from PacBio CCS 
Du X et al / Robust SV Benchmark of An Asian using long-sequencing

730 Figure S7 The size distributions for 0 1000bp and 1000 10000bp ranges for candidate SV

731 calls from ONT

732 Figure S8 The size distributions for 0 1000bp and 1000 10000bp ranges for candidate SV

733 calls from Bionano

734 Figure S9 Dot plot showed the synteny between assembled contigs from PacBio CCS reads

735 and the hs37d5 reference genome

736 Figure S10 Size distributions for 0 1000bp and 1000 10000bp ranges for candidate SV

737 calls from PacBio CCS assembly

738 Figure S11 The relationship between chromosome length and the number of SVs

739 Figure S12 The number of unique SVs and SVs overlapping among different candidate SV

740 callsets

741 Figure S13 Distribution of PCR Amplification results in different length ranges

742 Figure S14 Consistency rates of SV type, SV length, and breakpoint position between

743 Sanger sequenced variants and high-confidence SV calls with uncertain sites not excluded

744 Figure S15 Consistency rates of SV type, SV length, and SV position between Sanger

745 sequenced variants and high-confidence SV calls in different length ranges. Uncertain sites

746 were not excluded

747 Figure S16 Comparison of the Asian CNGB030001 benchmark and the GIAB HG002

748 benchmark within the $1.33 \mathrm{~Gb}$ overlapping benchmark regions.

749 Figure $\mathbf{S 1 7}$ The size distributions for 50 1000bp and > 1kb ranges for common and Asian

750 unique SVs by comparing GIAB benchmark to the Asian CNGB030001 benchmark.

751 Figure S18 GO enrichment of Asian-benchmark-specific SVs.

752 Table S1 Counts of identified high-confidence SVs on each chromosome

753 Table S2 Trio binning of CCS reads

754 Table S3 Counts of overlapping SVs from NGS platforms in comparison to the SV

755 benchmark 


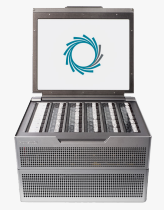

Oxford Nanopore
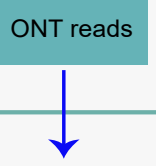

- - - - - - ,

'sniffles (ngmlr)

ONT_SV

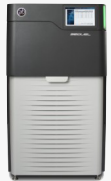

PacBio SMRT

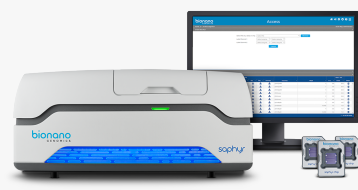

Bionano Irys

Bionano

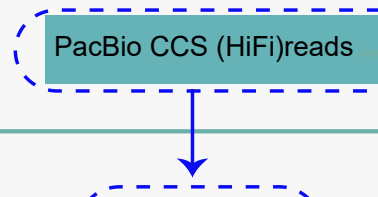

ípsv(minimap2)

'́pbsv(minimap2)!

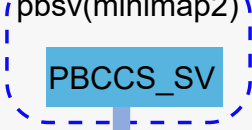

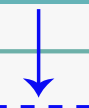

- - - - - - v

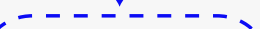

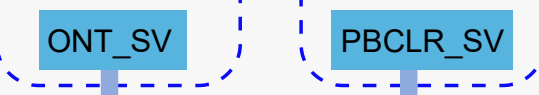

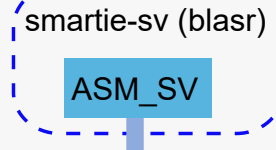

I

I

\section{BN_SV}

Combine five detected SVs datasets:

breakpoints range $<1000 \mathrm{bp}$ and length difference $<20 \%$

Filter SVs in the regions of high repeat content: telomere, centromere, segmental duplicate, tandem repeat

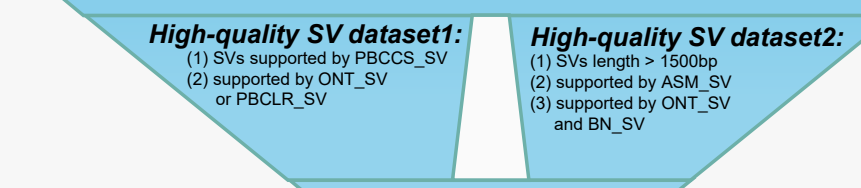

Validation

random selecte 544 SVs, and design primers on two sidesof the breakpoints

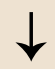

PCR amplication

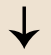

sanger sequencing of PCR products

High qualtiy of SVs:

merge two high-quality

SV datasets
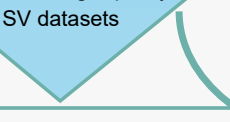

\section{Benchmark}

trio-binning PacBio CCS $(\mathrm{HiFi})$ reads

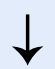

haplotype assembly

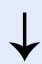

detect haplotype SVs $\rightarrow$ evaluation of different platforms and sequencing depth

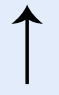

determine continuous high confident regions (CHCRs) 

chr8:51224---
951
961
971
chr8:51228---

321

331

341

351

361

Ref TATTTCTCCAATAATCTTGATATGAAATTTTGGTATAATTTTTTAAAATT"CTAGATAAAA --- GAAAAACATA'CTAATACTAAGGATGCAAACCATCAAAGCTCTGCTATTGTTATTCCCACC

Alt TATTTCTCCAATAATCTTGATATGAAATTTTGGTATAATTTTTTAAAATT

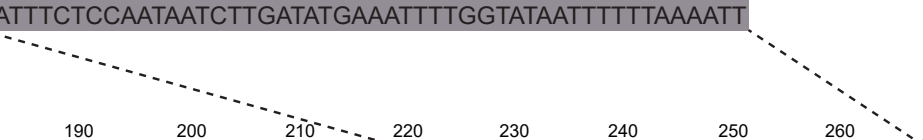

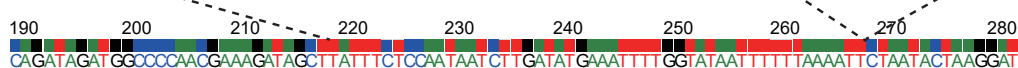

CTAATACTAAGGATGCAAACCATCAAAGCTCTGCTATTGTTATTCCCACC

CAAACCAT CAAAGCTCTGCTATTGTTATTCCCACCATGAACACCTGCACTGCAATCAAGCACAATT CACAGATGT GA

\section{Sanger}

Sequencing

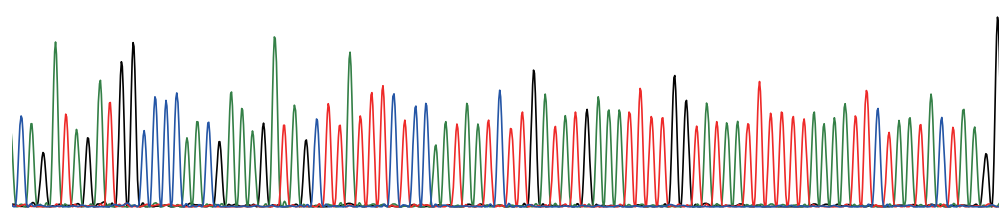

1

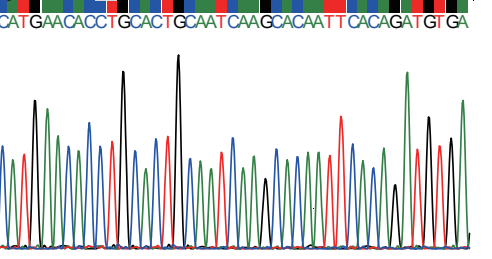

PCR products for dbsv59251

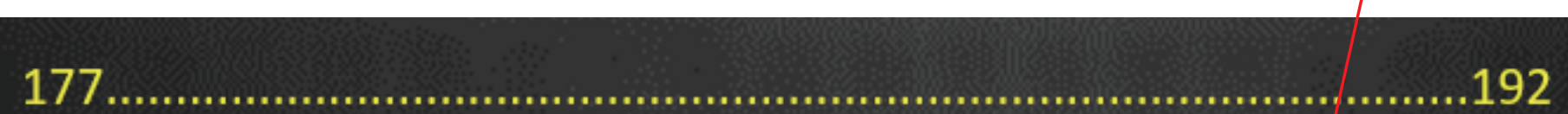

PCR

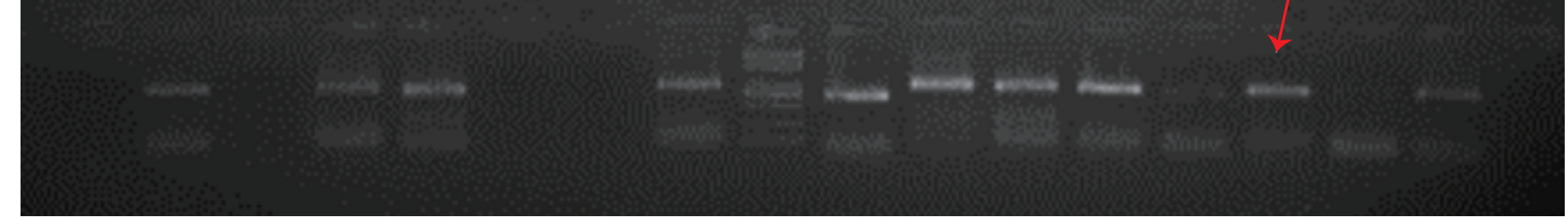

B

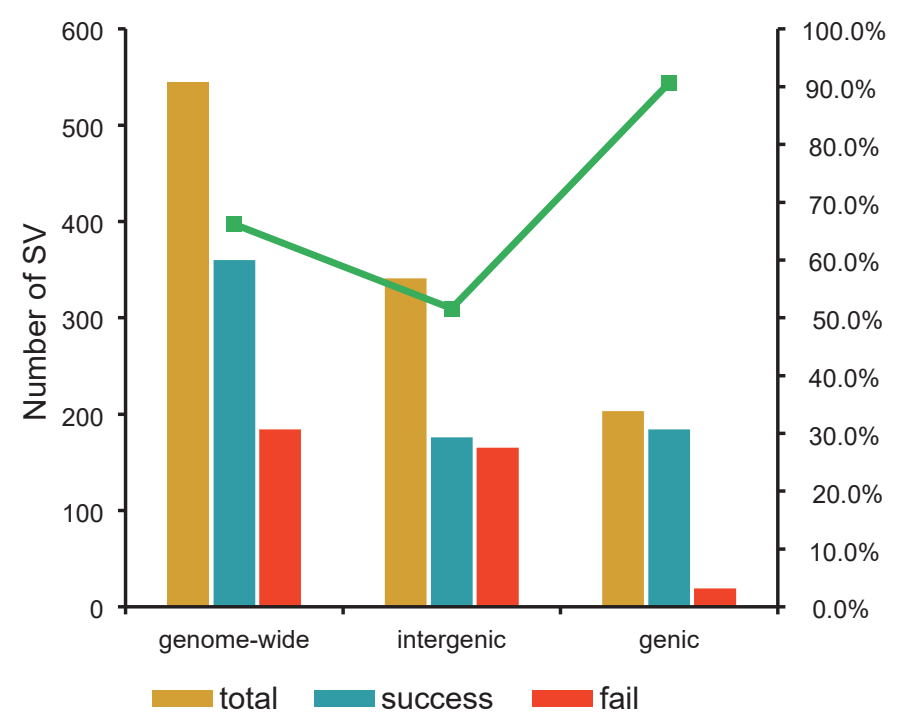

C

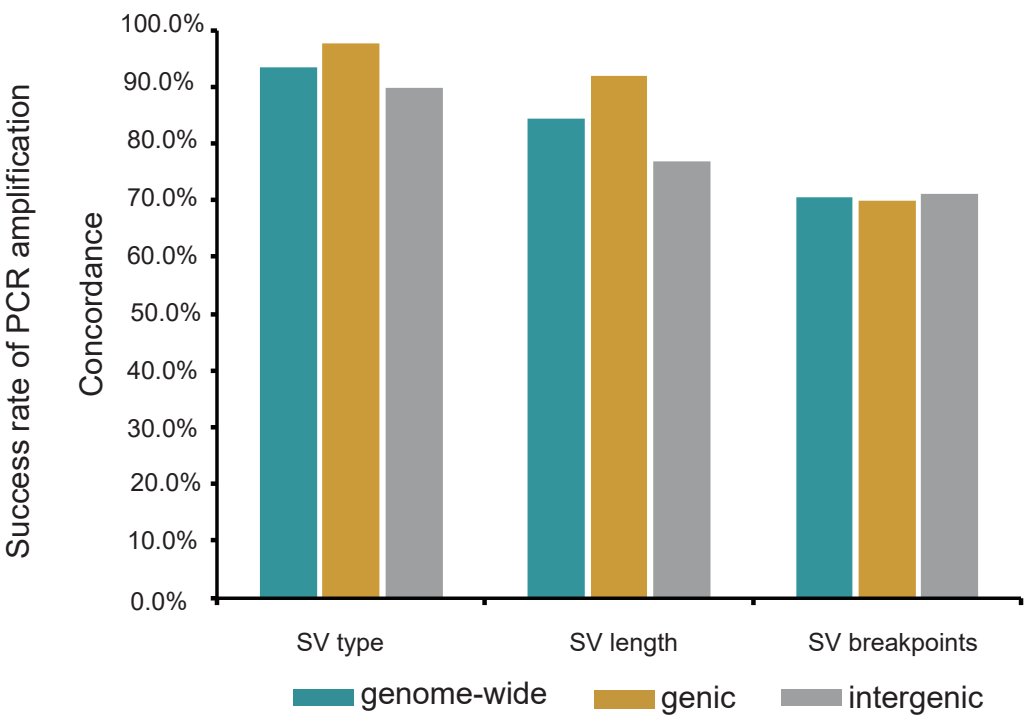


continuous high

confident regions

Maternal SVs

Paternal SVs
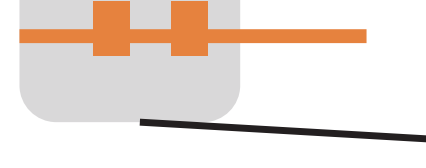

continuous high confident regions

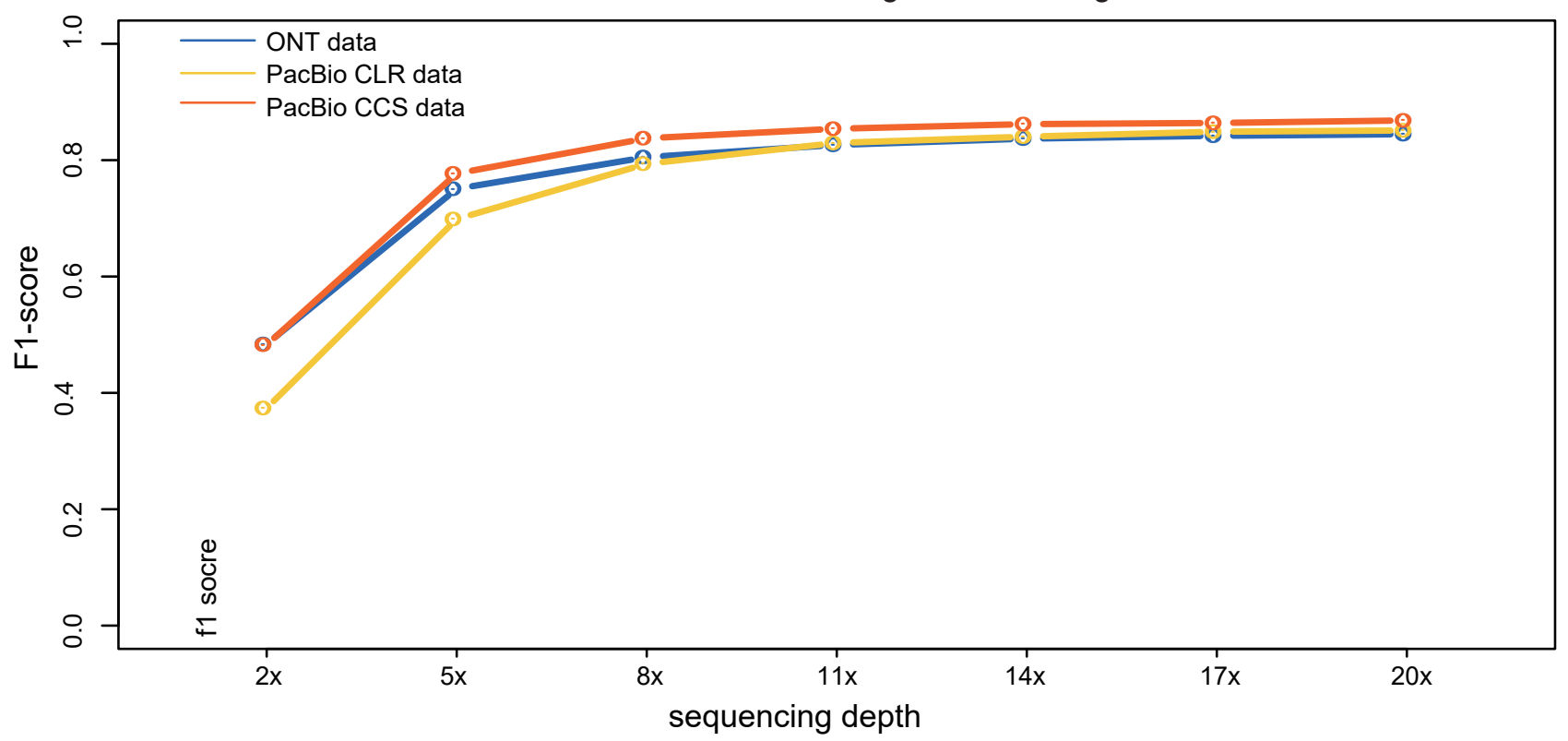


Table 1 Summary of sequencing results for different platforms

\begin{tabular}{llllllll}
\hline Platform & $\begin{array}{l}\text { Cell } \\
\text { num }\end{array}$ & $\begin{array}{l}\text { Sequencing } \\
\text { type }\end{array}$ & $\begin{array}{l}\text { Total } \\
\text { bases } \\
(\mathbf{G b p})\end{array}$ & $\begin{array}{l}\text { Depth } \\
(\mathbf{X})\end{array}$ & $\begin{array}{l}\text { Read length } \\
\text { (avg. } \pm \text { s.d., } \\
\text { bp) }\end{array}$ & $\begin{array}{l}\text { Read } \\
\text { accuracy }\end{array}$ & $\begin{array}{l}\text { GC content } \\
(\%)\end{array}$ \\
\hline $\begin{array}{l}\text { PacBio } \\
\text { CLR }\end{array}$ & 31 & subreads & 326.98 & 109 & $9,212 \pm 6,984$ & $0.8 \dagger$ & $42.66 \pm 5.82$ \\
PacBio & 24 & CCS & $869.48 ;$ & $266 ;$ & $11,961 \pm 3,662$ & $0.987 \pm 0.019$ & $40.59 \pm 5.63$ \\
CCS & & & $71.85^{*}$ & $22 X^{*}$ & & & \\
Oxford & 8 & 1D2 & 312.77 & $104 X$ & $24,589 \pm$ & $0.849 \pm 0.028$ & $40.79 \pm 5.55$ \\
Nanopore & & & & & 22,597 & & - \\
BioNano & 1 & BspQ1 & 341.67 & $114 \mathrm{X}$ & - & & \\
\hline
\end{tabular}

Note: * Total bases and depth after error correction for Circular Consensus Sequence (CCS). $\dagger$ Base quality of Pacbio CLR subreads produced by newer PacBio Sequel Sequencer is not available, so that we use an empirically overall read quality of 0.8 . 
Table 2 Insertion and deletion variation for different calling approaches

\begin{tabular}{|c|c|c|c|c|c|c|c|c|c|}
\hline \multirow[b]{2}{*}{ Data } & \multirow[b]{2}{*}{$\begin{array}{l}\text { Calling } \\
\text { Methods }\end{array}$} & \multicolumn{4}{|l|}{ DEL } & \multicolumn{4}{|l|}{ INS } \\
\hline & & Count & $\begin{array}{l}\text { Min } \\
\text { length } \\
\text { (bp) }\end{array}$ & $\begin{array}{l}\text { Max } \\
\text { length (bp) }\end{array}$ & $\begin{array}{l}\text { Average } \\
\text { length } \\
\text { (bp) }\end{array}$ & Count & $\begin{array}{l}\text { Min } \\
\text { length } \\
\text { (bp) }\end{array}$ & $\begin{array}{l}\text { Max } \\
\text { length } \\
\text { (bp) }\end{array}$ & $\begin{array}{l}\text { Average } \\
\text { length } \\
\text { (bp) }\end{array}$ \\
\hline $\begin{array}{l}\text { Pacbio } \\
\text { CLR }\end{array}$ & $\begin{array}{l}\text { minimap2 } \\
+ \text { pbsv }\end{array}$ & 5,871 & 50 & 45,516 & 562 & 6,936 & 50 & 10,273 & 403 \\
\hline $\begin{array}{l}\text { Pacbio } \\
\text { CCS }\end{array}$ & $\begin{array}{l}\text { minimap2 } \\
+ \text { pbsv }\end{array}$ & 8,317 & 50 & 75,746 & 480 & 9,584 & 50 & 9,805 & 470 \\
\hline ONT & $\begin{array}{l}\text { ngmlr+sni } \\
\text { ffles }\end{array}$ & 7,668 & 50 & $62,462,724$ & 90,997 & 6,717 & 50 & 7,750 & 326 \\
\hline BioNano & $\begin{array}{l}\text { Electronic } \\
\text { Mapping }\end{array}$ & 1,517 & 224 & $4,407,162$ & 65,053 & 3,241 & 231 & $\begin{array}{l}970,56 \\
7\end{array}$ & 6,404 \\
\hline $\begin{array}{l}\text { CCS } \\
\text { Assembly }\end{array}$ & $\begin{array}{l}\text { Blasr+sm } \\
\text { artie-sv }\end{array}$ & 10,345 & 50 & 75,030 & 788 & 17,382 & 50 & 64,449 & 611 \\
\hline
\end{tabular}

